\title{
Negative regulator of stem cell-based bone marrow regeneration
}

\author{
Huili Lu ${ }^{1}$, Wenwei Mao ${ }^{1}$, Shunying Zhu ${ }^{1}$, Lan Qian ${ }^{1}$, Zhonghui Zhang ${ }^{1}$, Mingyuan $\mathrm{Wu}^{1}$, Qun Wang ${ }^{1}$, Wei $\operatorname{Han}^{1}$ \\ ${ }^{1}$ Laboratory of Regenerative Medicine, School of Pharmacy, Shanghai Jiao Tong University, Shanghai, China
}

Monokine induced by IFN- $\gamma$ (Mig) is also called CXCL9. It is a CXC-chemokine and reported to have myelosuppressive activity just as many other CXC-chemokines. Murine mig was identified in our large scale gene expression array screening for candidate regulators of bone marrow (BM) regeneration. We hypothesized that Mig could suppress the proliferation of hematopoietic stem/progenitor cells in vivo, through which it may protect BM from cancer chemotherapy drugs such as 5-fluorouracil (5-Fu) that causes BM damage as its major dose-limiting and life-threatening side effect. The recombinant murine Mig (rMuMig) administrated to normal mice was demonstrated to reduce the number of BM cells significantly by inhibition of G1-to-S phase transition of the cells. When the protein was injected into the mice before 5-Fu, it protected up to $60 \%$ of the mice from dying of high dose 5-Fu, compared to $10 \%$ of the PBS control. Furthermore, the BM hematopoiesis of the rMuMig treated mice recovered significant quicker than the control group according to the peripheral blood cell counts, BM cell counts, and the BM histology. We further investigated the mechanisms of myeloprotective role of rMuMig. FACS analysis revealed that rMuMig inhibited BM cells cycling into S-phase. The endogenous Mig and its receptor CXCR3 expression were up-regulated after 5-Fu treatment of the mice, indicating the Mig/CXCR3 signaling pathway is a natural hematopoietic protective mechanism. Neutralization the endogenous Mig with anti-Mig antibody accelerated the recovery of BM and increased the mice survival rate after 5-Fu treatment. In conclusion, we have identified that Mig/CXCR3 is an endogenous BM protection system. By mechanismbased proper use of the natural BM protective system, we demonstrate that rMuMig as well as rHuMig (recombinant human Mig) deliver the BM protection against the BM suppression side effect of cancer chemotherapy. Our studies suggest that Mig may be developed as a new chemo-protecting drug and may contribute greatly to the improvement of cancer therapy in combination with chemotherapy.

Keywords: CXCL9, Mig, bone marrow, hematopoiesis, chemotherapy

Cell Research (2008) 18:s95. doi: 10.1038/cr.2008.185; published online 4 August 2008

Correspondence: Wei Han

Tel/Fax: 021-3420-4750

E-mail:weihan@sjtu.edu.cn

$\mathrm{PhD}$, Professor, Biomedical Sciences Head, Regenerative Medicine Laboratory, School of Pharmacy, Associate Director, Shanghai Stem Cell Institute, Shanghai Jiaotong University, 800 DongChuan Road, Shanghai 200240, China

After graduated from Qingdao Medical College and received his Master degree in Histology \& Embryology in Shanghai Medical University, he received his PhD in biomedical sciences from Mount Sinai School of Medicine in New York. In 1994, he joined Malcolm Moore's Lab at Memorial Sloan-Kettering Cancer Center in New York, worked in the field of hematopoietic stem cells (HSCs). Cloned and produced the first human Notch ligand- human Delta-like-1(hDll1), a critical self-renewal factor of HSCs. In 1999, he moved to the Biotech industry. He co-invented the high efficient ( $>95 \%)$ gene transfer methods to human hematopoietic stem cell and lymphocytes, and is one of the major contributors to the world first clinical grade Lentivirus Vector Transduced Lymphocyte Product, that was successfully used in Phase I and II clinical trials treating HIV patients. Dr Han joined Shanghai Jiaotong University in Jan. 2004, head the research group focusing on Regenerative Medicine. His group has identified 128 genes regulating bone marrow (BM) regeneration. Two of them were proven to be potent $\mathrm{BM}$ protector against the chemotherapy induced $\mathrm{BM}$ damage. 